\title{
Merkel cell carcinoma: A series of seven cases
}

\author{
Yong Woo Lee ${ }^{1}$, Yong Chan Bae ${ }^{1,2}$, Su Bong Nam ${ }^{1}$, Seong Hwan Bae ${ }^{1}$, Hoon-Soo Kim ${ }^{3}$ \\ ${ }^{1}$ Department of Plastic and Reconstructive Surgery, Pusan National University School of Medicine, Busan; ${ }^{2}$ Biomedical Research Institute, \\ Pusan National University Hospital, Busan; ${ }^{3}$ Department of Dermatology, Pusan National University School of Medicine, Busan, Korea
}

Background Merkel cell carcinoma (MCC) is a rare neuroendocrine malignancy affecting the skin, for which timely diagnosis and aggressive treatment are essential. MCC has most often been reported in Caucasians, and case reports in Asians are rare. This study presents our experiences with the surgical treatment and radiotherapy of MCC in Asian patients.

Methods We retrospectively reviewed the records of seven MCC patients between 2000 and 2018 from a single institution, and analyzed patient characteristics, tumor characteristics, surgical treatment, sentinel lymph node evaluation, reconstruction, adjuvant radiation therapy, and prognosis.

Results Eight MCC lesions occurred in seven patients, most commonly in the head and neck region. All patients underwent surgical excision with reconstruction. The final surgical margin was $1.0 \mathrm{~cm}$ in most cases, and reconstruction was most commonly performed with a splitthickness skin graft. Five patients received adjuvant radiotherapy, and two patients received sentinel lymph node biopsy. During the follow-up period, three patients remained well, two died from other causes, one experienced recurrence, and one was lost to follow-up.

Conclusions We treated seven Asian MCC patients and our series confirmed that MCC is a very dangerous cancer in Asians as well. Based on our experiences, thorough surgical excision of MCC with histopathological clearance should be considered, with sentinel lymph node evaluation if necessary, followed by appropriate reconstruction and careful postoperative observation. Adjuvant radiation therapy is also recommended for all Asian MCC patients. The results of this case series may provide guidance for the treatment of Asian MCC patients in the future.

Keywords Neoplasms / Carcinoma, Merkel cell / Margins of excision / Mohs surgery / Prognosis
Correspondence: Yong Chan Bae Department of Plastic and Reconstructive Surgery, Biomedical Research Institute, Pusan National University Hospital, Pusan National University School of Medicine, 179 Gudeok-ro, Seo-gu, Busan 49241, Korea

Tel: +82-51-240-7273

Fax: +82-51-243-9405

E-mail: baeyc2@hanmail.net

This work was supported by a clinical research grant from Pusan National University Hospital in 2019.

This article was presented at PRS Korea 2018 on November 9-11, 2018, in Seoul, Korea.

Received: April 5, $2019 \bullet$ Revised: August 5, $2019 \bullet$ Accepted: August 6, 2019

pISSN: 2234-6163 • elSSN: 2234-6171 • https://doi.org/10.5999/aps.2019.00465• Arch Plast Surg 2019;46:441-448

\section{INTRODUCTION}

Merkel cell carcinoma (MCC) is a neuroendocrine trabecular cancer of the dermis, first described by Toker in 1972, that poses a significant risk of local invasion, lymph node metastasis, and distant metastasis. It is rare, but is primarily found in older Caucasian patients [1]. MCC is very aggressive; its mortality rate exceeds 33\%, making it twice as lethal as malignant melanoma $[1,2]$. The elderly and immunocompromised persons are at particularly high risk [3]. As mentioned earlier, MCC is more common in Caucasian patients, and its incidence varies across different regions of the world $[4,5]$. According to the most recent available analysis, based on the 2011 Surveillance, Epidemiology, and End Results data in the United States, the inci- 
dence of MCC was 0.79 per 100,000. Of these cases, $94.9 \%$ were white, $1.0 \%$ black, and $4.1 \%$ belonged to other races (Asian-Pacific Islander, American Indian, or other), indicating that MCC was very rare in Asian patients [6]. Although the reported incidence of MCC has increased by $8 \%$ per year between 1986 and 2001, cases in Asian patients have been rarely reported [7]. A 2008 study presented 16 Japanese MCC patients, a 2010 study described seven Korean patients, and a 2012 study analyzed 22 Chinese patients [8-10]. However, no case studies of $\mathrm{MCC}$ in Asian patients have been reported since.

For more than 20 years, wide excision with tumor-negative resection margins and adjuvant radiotherapy (RT) of the primary tumor site and regional lymph node bed have been widely recommended as the treatment for early-stage MCC [11]. We retrospectively reviewed the clinical outcomes of seven Korean MCC patients who underwent surgical excision and adjuvant RT between 2000 and 2018 at a single institution. By sharing our experiences with rare cases of MCC in Asia, we hope to help plastic surgeons who are not familiar with this disease to refer MCC patients appropriately, increasing the likelihood of successful outcomes.

\section{METHODS}

This study reviewed and analyzed the medical records of seven patients who were diagnosed with MCC between 2000 and 2018. The characteristics of each patient (age and sex), the tumors (location and number of lesions), surgical treatment (surgical excision and reconstruction), lymph node evaluation, adjuvant RT, and prognosis were analyzed.

The surgical treatments included wide local excision (WLE), Mohs micrographic surgery (MMS), and modified MMS. Our analysis investigated the frequency of each method and the final surgical margin. WLE is an extensive excisional procedure with a 1- to 2-cm surgical resection margin that can be used for MCC treatment according to the guidelines of the National Comprehensive Cancer Network (NCCN). In MMS, an average of 2-4 $\mathrm{mm}$ margin of clinically normal surrounding tissue is excised, and the procedure is continued until the absence of cancerous tissue is confirmed throughout the excision. Modified MMS combines the principles of MMS and WLE. First, WLE is performed as described above. In addition, a new resection margin is drawn with a width of $1-2 \mathrm{~mm}$ within the previous resection margin. The area between the previous resection margin and the new resection margin is divided into approximately 10 to 20 sections. The base of the lesion is also divided into sections, if necessary. All sections are then mapped and resected in order. The resected section tissues are sent for frozen biopsy. If the fro- zen biopsy results show malignancy, additional excision from that section is performed with the same procedure until cancer cells are no longer visible on the frozen biopsy.

In order to preserve the surrounding normal tissue as much as possible for functional and cosmetic reasons, tumors in some parts of the face are excised with a surgical resection margin of 3-4 $\mathrm{mm}$ from the area containing the malignancy. Tumors in other regions, such as the upper or lower extremity, are excised with a surgical resection margin of $0.5-1 \mathrm{~cm}$ from the area containing the malignancy.

The reconstruction methods for the defects included local flaps, split-thickness skin grafts (STSG), and free flaps.

The preoperative examinations in patients requiring lymph node evaluation included physical examination, lymphangiography, computed tomography, and positron emission tomography-computed tomography (PET-CT). The preoperative examinations confirmed the location of the sentinel lymph node (SLN). SLN biopsy (SLNBx) or core needle biopsy was then performed, if necessary. Elective lymph node dissection (ELND) was also performed if malignancy was found on SLNBx or core needle biopsy.

Adjuvant RT is recommended in the NCCN guideline for MCC patients with tumors exceeding $1.5 \mathrm{~cm}$ in size, perineural or vascular invasion, microscopic positive findings at the margin, residual disease, and/or regional lymph node involvement [12].

Prognosis was assessed in terms of survival, recurrence, and loss to follow-up after treatment.

MCC staging was carried out using the eighth edition of the American Joint Committee on Cancer staging system.

We conducted this study after receiving approval from the Institutional Review Board (IRB No. H-1905-016-079). Informed written consent was obtained from all patients.

\section{RESULTS}

\section{Patient and tumor characteristics}

Of the seven patients, two were men and five were women, ranging in age from 56 to 86 years (mean age, 70 years). Of the seven patients, one patient had two scalp lesions, and the remaining patients had one lesion each. Therefore, the seven patients had a total of eight lesions. The most common tumor site was the head and neck region (four patients), followed by the lower extremities (two patients), and the upper extremities (one patient). The patients were observed for 1 month to 14 years after surgery (Table 1). 
Table 1. Characteristics of patients and their surgical treatment, lymph node evaluation, adjuvant RT, and prognosis

\begin{tabular}{|c|c|c|c|c|c|c|c|c|c|c|}
\hline $\begin{array}{c}\text { Case } \\
\text { no. }\end{array}$ & $\begin{array}{c}\text { Sex/age } \\
\text { (yr) }\end{array}$ & Location & $\begin{array}{l}\text { Surgical } \\
\text { method }\end{array}$ & $\begin{array}{c}\text { Surgical } \\
\text { margin }(\mathrm{cm})\end{array}$ & Reconstruction & SLNBx & $\begin{array}{c}\mathrm{LN} \\
\text { dissection }\end{array}$ & $\begin{array}{c}\text { Adjuvant } \\
\text { RT }\end{array}$ & Prognosis & Staging \\
\hline 1 & $M / 82$ & Scalp (two lesions) & WLE & $1.5,0.5$ & STSG, local flap & No & No & No & Death & $\|$ \\
\hline 2 & $\mathrm{~F} / 76$ & Face & Modified MMS & 1 & Local flap & No & No & Yes & Loss to follow-up & I \\
\hline 3 & F/86 & Extremities & Modified MMS & 3 & STSG & No & No & No & Death & I \\
\hline 4 & $\mathrm{~F} / 71$ & Face & MMS & - & STSG & No & No & Yes & Survival (14 mon) & I \\
\hline 5 & $\mathrm{~F} / 56$ & Extremities & Modified MMS & 1 & STSG & Yes & Yes & Yes & Survival (9 yr) & III \\
\hline 6 & $F / 63$ & Face & Modified MMS & $0.5-1$ & Free flap & Yes & No & Yes & Survival (14 yr) & I \\
\hline 7 & $M / 58$ & Extremities & Modified MMS & 1 & STSG & No & Yes & Yes & $\begin{array}{l}\text { Recurrence (1 mon } \\
\text { postoperatively) and } \\
\text { metastasis ( } 8 \text { mon } \\
\text { postoperatively) }\end{array}$ & III \\
\hline
\end{tabular}

RT, radiotherapy; SLNBx, sentinel lymph node biopsy; LN, lymph node; M, male; F, female; WLE, wide local excision; STSG, split-thickness skin graft; MMS, Mohs micrographic surgery.

\section{Surgical treatment}

All patients underwent surgical excision as the primary treatment. Modified MMS was performed in five patients, WLE in one patient, and MMS in one patient. The final surgical resection margin was $1.0 \mathrm{~cm}$ for four lesions, followed by $0.5 \mathrm{~cm}$ for two lesions, $1.5 \mathrm{~cm}$ for one lesion, and $3 \mathrm{~cm}$ for one lesion. After excision, skin coverage for the defects was performed using STSG for five lesions, a local flap for two lesions, and a free flap for one lesion. The local flaps included one simple advancement flap and one rhomboid flap, and the free flap was a radial forearm free flap (Table 1).

\section{Lymph node evaluation}

Not all patients underwent lymph node evaluation. SLNBx was performed in two patients, with one negative finding and one positive finding. ELND was performed in the patient with a positive finding. One patient with recurrent MCC showed a palpable axillary lymph node on physical examination and underwent ELND after core needle biopsy of the axillary lymph node showed a positive finding (Table 1).

\section{Adjuvant RT}

Five patients underwent adjuvant RT after surgery. One of the other two patients planned to undergo RT, but died 1 month after surgery. The other patient did not undergo RT due to a poor general condition (Table 1).

\section{Prognosis}

Three of the seven patients survived without recurrence, two died, one experienced recurrence, and one was lost to follow-up. Two of the three survivors had no particular problems for 6 years or more postoperatively, and the other survivor remained well at 14 months postoperatively. Two patients died at 1 month and 3 months after the operation, respectively, due to aspiration pneumonia, a disease not related to MCC. One patient received palliative care due to recurrence and metastasis of MCC. One patient was lost to follow-up after 2 months postoperatively (Table 1).

\section{Cases}

Case 1

An 82-year-old male patient had two scalp lesions that were initially diagnosed as basal cell carcinoma. The two lesions were treated with WLE, with surgical margins of $1.5 \mathrm{~cm}$ (anterior lesion) and $0.5 \mathrm{~cm}$ (posterior lesion). After excision of the lesions, the anterior defect was reconstructed with STSG and the posterior defect with a simple advancement local flap. Both resected lesions were diagnosed as MCC based on histopathological findings. The patient died due to aspiration pneumonia 1 month after surgery, and the scheduled RT was not performed.

\section{Case 2}

A 76-year-old female patient underwent excision surgery for a nodule on the left malar region at the department of general surgery at a local hospital, and the nodule was diagnosed as MCC. The patient underwent surgical excision with modified MMS with a $1-\mathrm{cm}$ surgical margin at the previous MCC excision site, and no tumor was found in the first session. Then, she received reconstruction with a rhomboid flap. Adjuvant RT was performed at the left malar region ( $50 \mathrm{~Gy}, 28$ times) as a prophylactic treatment after surgery. After 2 months postoperatively, the patient was lost to follow-up.

\section{Case 3}

An 86-year-old female patient underwent modified MMS with a 3-cm surgical resection margin for MCC on the left thigh, and no tumor was found in the first session. The defect was reconstructed with STSG. Adjuvant RT was not performed due to 
the patient's age and poor general condition. She died due to aspiration pneumonia at 3 months postoperatively.

\section{Case 4}

A 71-year-old female patient underwent MMS for MCC on the glabellar region, and the defect was reconstructed with STSG. After the operation, the patient was treated with adjuvant RT (54 Gy, 27 times) and remained well at 14 months after surgery (Fig. 1).

\section{Case 5}

A 56-year-old female patient underwent modified MMS with a 1 -cm surgical margin for MCC on the left knee including exposed fascia, and no tumor was found in the first session.
Lymph node dissection was performed after a positive finding on SLNBx at the left inguinal region. Adjuvant RT was performed at the left knee (50 Gy, 28 times), left inguinal area, and external iliac lymph node area (54 Gy, 30 times). She remained well at 9 years postoperatively (Fig. 2).

\section{Case 6}

A 63-year-old female patient underwent modified MMS for MCC on the left nasolabial fold. In the first session, three 2-mmwide sections (sections 1-3), which were mapped along the 0.5 $\mathrm{cm}$ surgical resection margin, were divided. Then, six sections (sections 4-9) with the same width along the 1-cm surgical resection margin were also mapped. Frozen biopsy during the operation showed no tumor findings in sections 3-9, but positive

\section{Fig. 1. Case 4}

A 71-year-old woman with a Merkel cell carcinoma lesion in the glabellar region. (A) Before Mohs micrographic surgery (MMS). (B) After MMS. (C) Postoperative photo obtained 12 months after reconstruction (split-thickness skin graft).
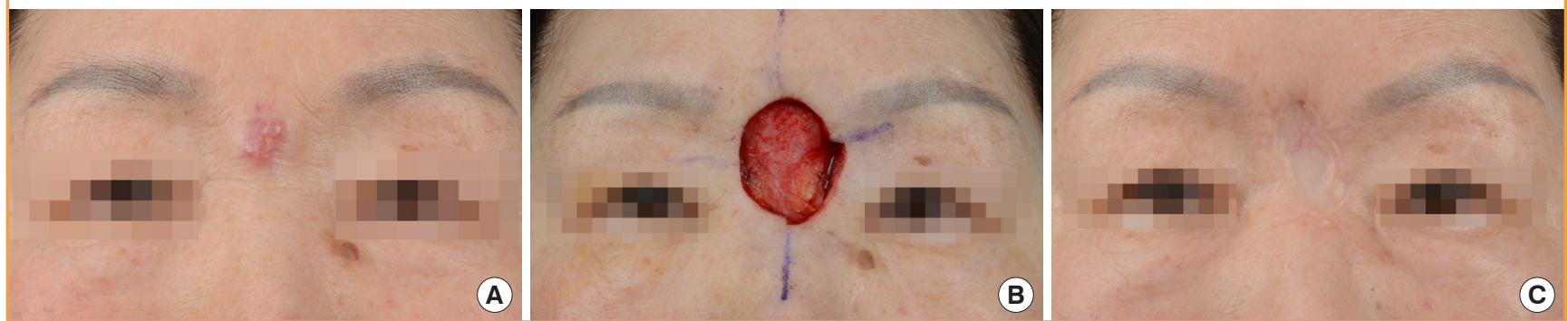

\section{Fig. 2. Case 5}

A 56-year-old woman with Merkel cell carcinoma on the left knee. (A) Before modified Mohs micrographic surgery (MMS). (B) After modified MMS and reconstruction (split-thickness skin graft). (C) Postoperative photo obtained 6 years after surgery.
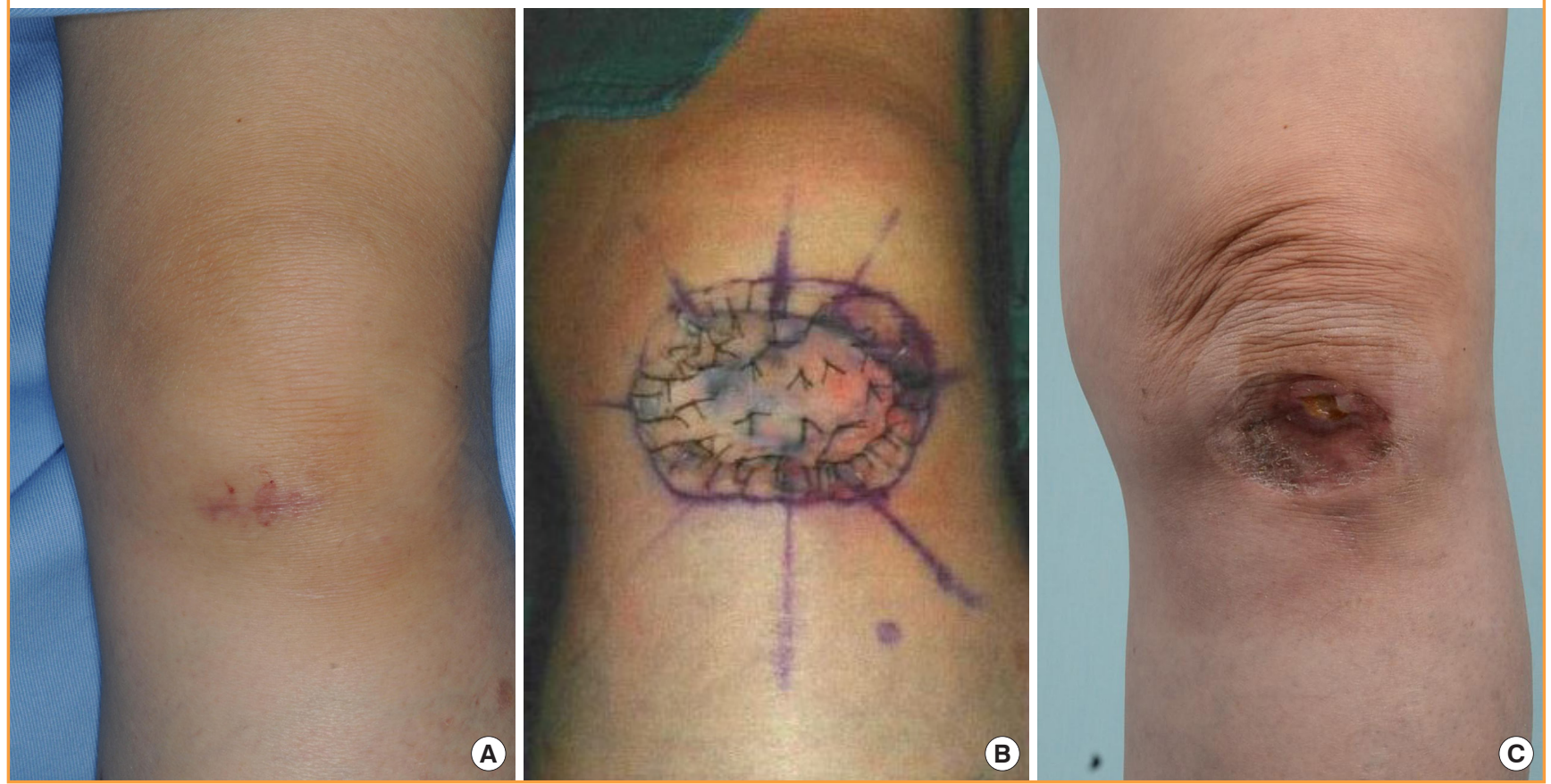
findings were seen in sections 1 and 2 . Thus, additional excision was performed $1 \mathrm{~mm}$ inward from the surgical resection margin of the first and second sections at a distance of $3 \mathrm{~mm}$. No tumor findings were seen in the second session. Radial forearm free flap reconstruction for the defect was performed. SLNBx at the left submandibular region showed negative findings. Adjuvant RT was done at the left nasolabial fold (66 Gy, 33 times) and the left neck (57 Gy, 30 times). The patient remained well at 14 years postoperatively (Fig. 3).

\section{Case 7}

A 58-year-old male patient underwent modified MMS with a 1 -cm surgical margin for MCC on the right shoulder and no tumor was found in the first session. The defect was reconstructed with STSG.

At 1 month after surgery, recurrence was found. In addition, a core needle biopsy was performed due to a palpable lymph node at the right axillary region, with positive findings. Modified MMS was performed again with a $1-\mathrm{cm}$ surgical resection margin for the recurrent mass, and no tumor was found in the first session. The defect was reconstructed with STSG. Lymph node dissection was performed at the right axillary region. Adjuvant RT was performed on the right shoulder (56 Gy, 28 times) and the right axillary lymph node region (60 Gy, 30 times) after the second operation.

However, the patient refused chemotherapy due to the presence of multiple rib bone metastases on chest CT at 8 months postoperatively, and the patient was transferred to palliative care (Fig. 4).

\section{DISCUSSION}

MCC tends to occur in older Caucasians; in fact, more than $90 \%$ of MCC patients have been reported to be Caucasian, and

\section{Fig. 3. Case 6}

A 63-year-old woman with Merkel cell carcinoma on the left nasolabial fold. (A) Before modified Mohs micrographic surgery (MMS). (B) Mapping for modified MMS. (C) After modified MMS. (D) Postoperative photo obtained 6 years after surgery.
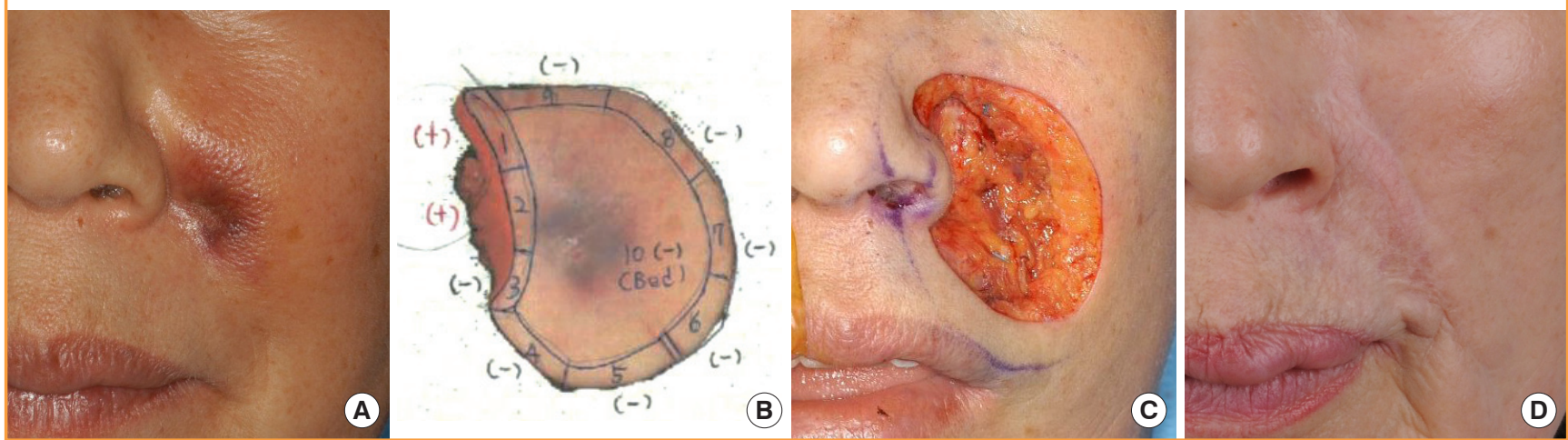

\section{Fig. 4. Case 7}

A 58-year-old man with Merkel cell carcinoma (MCC) on the right shoulder. (A) Before modified Mohs micrographic surgery (MMS) for primary MCC. (B) Mapping for modified MMS for primary MCC. (C) After modified MMS for primary MCC. (D) Before modified MMS for recurrent MCC. (E) Mapping for modified MMS for recurrent MCC.
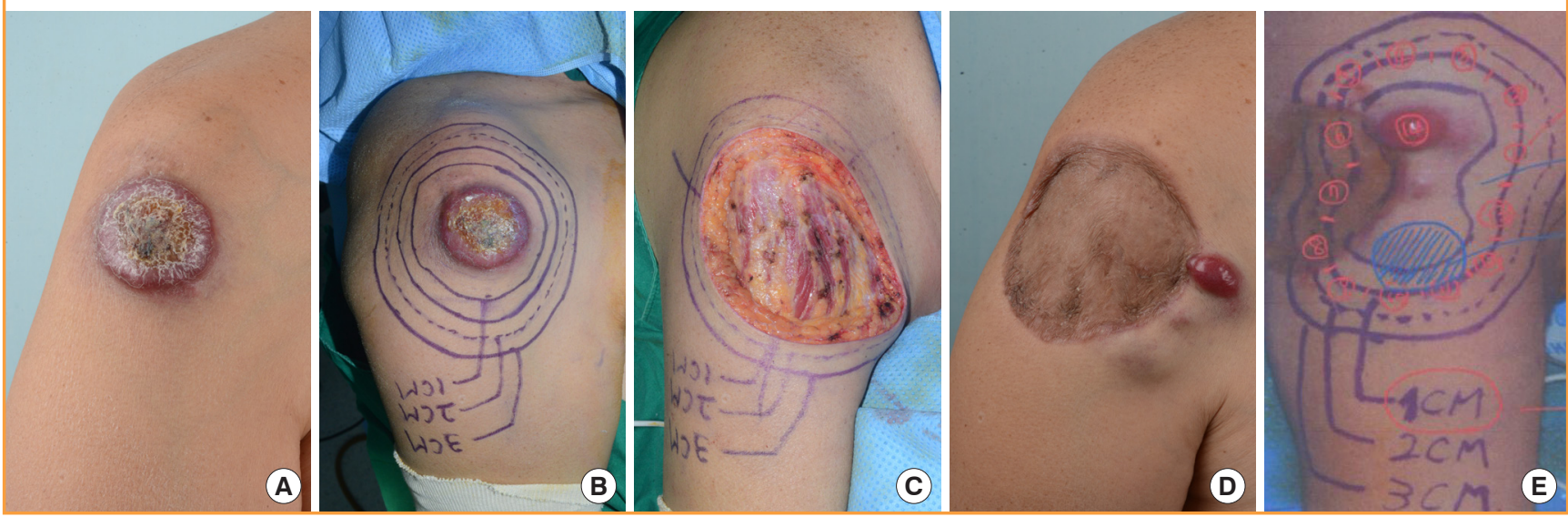
few reports have described Asian MCC patients [7]. Therefore, although this study includes relatively few cases, it is one of the few studies on Asian MCC patients.

Although the clinical features of early-stage MCC are unclear, MCC may present as erythematous or violaceous papules and nodules, and it is generally diagnosed by histologic and immunohistochemical findings. Most MCCs occur at the head and neck region or at the extremities, in accordance with the findings of this study [13].

As a standard treatment option for stage I and II MCC, margin-negative local excision is performed and surgical nodal evaluation is usually considered with SLNBx. If positive lymph nodes are found, complete nodal dissection may be considered, and the patient is upstaged to stage III. Adjuvant RT may be considered if there are concerns about the adequacy of the surgical resection margin for the primary tumor or if the nodal staging process is incomplete. Stage II MCC standard treatment options include margin-negative local excision followed by a SLN procedure and adjuvant RT. Chemotherapy may be considered for stage IV MCC patients, but insufficient evidence exists regarding whether chemotherapy results in permanent disease control or regional palliation. If chemotherapy is not an appropriate option for a patient with stage IV MCC, surgery and/ or radiation therapy may be considered for local or regional palliation [14].

The primary treatment of $\mathrm{MCC}$ is surgical excision [15]. According to the current NCCN guidelines for MCC treatment, WLE with a 1- to 2-cm surgical resection margin down to the fascia of the muscle or periosteum when clinically feasible is recommended for all primary MCC tumors [16]. However, different margins of excision have not yet been compared in any controlled clinical trials [9].

In the study of primary MCC tumors in a large population reported by Perez et al. [17], a 1-cm surgical resection margin did not show a significant association with an elevated risk of local recurrence, a decrease in overall survival, or a decrease in disease-specific survival. In this study, four patients (i.e., more than half of the patients) underwent surgical resection with a $1-\mathrm{cm}$ surgical resection margin. Two of them survived, one experienced local recurrence, and one was lost to follow-up. One lesion, at the lower extremity, had a surgical resection of $3 \mathrm{~cm}$, which exceeds the maximum surgical resection margin of $2 \mathrm{~cm}$ recommended by the NCCN. The only patient who underwent MMS in this study had no local recurrence, consistent with a report indicating that maximal local control can be achieved through MMS [18]. However, it is difficult to meaningfully compare our findings to those of previous studies due to the small number of patients.
In this study, modified MMS was performed in five patients, and was the main method for surgical excision. In this method, a plastic surgeon and a pathologist collaborate to perform margin-negative resection of cancer. If the cancer extends beyond the resected tissue, additional excision is repeated until no more cancer cells are present in the margin of the surgically resected tissue. Of the five patients who underwent modified MMS, two remained well for 6 years or more postoperatively and one remained well through 14 months of follow-up. One died from a disease not related to MCC, and the other one was lost to follow-up. The observation that over half of the patients who underwent modified MMS remained well, while another died from an unrelated disease, indicates that modified MMS was superior to other surgical excision methods, at least within the limitations inherent to this study because of its small sample size. Of the five patients who underwent modified MMS, three had MCC in the upper or lower extremity and two had MCC in the head and neck region. This indicates that modified MMS can be applied to various regions of the body.

WLE without a histological examination at the surgical resection margin during surgery can be performed quickly. Therefore, this procedure can be used in patients with a poor general condition who have difficulty undergoing general anesthesia, so that the surgery can be performed straightforwardly under local anesthesia. However, there is a risk of recurrence or metastasis since the surgical margin may be positive. MMS has the advantage of performing complete peripheral and deep histological margin control while maximally sparing normal skin close to the tumor, especially when performed in the head and neck region, which is a cosmetically sensitive structure. However, this process is slow, often taking several hours. Prospective clinical trials comparing WLE and MMS in MCC have not yet been performed, but retrospective studies of MMS have found it to be more effective than WLE $[14,19]$.

A number of clinical and pathologic factors have been associated with a poor prognosis in MCC including male sex [20], older age [21], increased tumor size [22], and an immunocompromised state. However, regional nodal status is the single most important prognostic characteristic of clinically localized MCC $[21,22]$. Not all patients in the study underwent lymph node evaluation because the decision to perform lymph node evaluation took into account each patient's general condition and preferences. Two patients underwent SLNBx and one underwent lymph node dissection with positive findings. Both patients who underwent SLNBx remained well without any problems for 6 years or more after surgery. SLNBx is performed to stage the lymph node basin because the most common location of metastasis of MCC is the draining lymph node basin $(27 \%-$ 
$60 \%$ ) followed by the distant skin (9\%-30\%), lung (10\%-23\%), central nervous system (18\%), bone (10\%-15\%) and liver (13\%). It is also possible to perform SLNBx in patients with clinically negative lymph nodes since roughly $20 \%$ to $30 \%$ of clinically negative MCC patients have positive SLN findings on histology. SLN mapping can be performed using lymphoscintigraphy, and the first-line treatment in the majority of patients with palpable lymphadenopathy and micrometastasis to the regional lymph nodes is complete lymph node dissection [23].

In the study, adjuvant RT was recommended to all seven patients, and most patients underwent adjuvant RT, with the exception of those who died beforehand or whose general condition was poor. Since MCC is highly sensitive to radiation, adjuvant $\mathrm{RT}$ is an important component of MCC treatment. $\mathrm{Al}$ though Allen et al. [13], in a retrospective review of 251 patients, reported that adjuvant RT did not significantly improve local control, most other clinical studies have shown that postoperative adjuvant RT improved local control $[24,25]$. Currently, adjuvant RT is recommended for patients with tumors larger than $1.5 \mathrm{~cm}$, vascular invasion, perineal invasion, microscopic positive margins, residual disease, and/or regional lymph node involvement [12]. According to the NCCN guidelines, adjuvant nodal RT is routinely recommended when SLNBx is not performed or if a patient shows clinically positive regional lymph node findings [12]. Nonetheless, a more specific consensus regarding the indications for adjuvant $\mathrm{RT}$ is needed.

MCC is a rare, but very aggressive, skin cancer. Prompt diagnosis and active treatment are very important for managing this disease. As one of the very few studies about MCC in Asian patients, we present an analysis of our experience managing seven Asian patients with MCC.

We performed thorough surgical excision for MCC, with histologically negative findings of cancer in the surgical resection margin. To do so, we used MMS or modified MMS. The SLN was evaluated if necessary, and lymph node dissection was performed in cases with positive lymph node findings. However, some of the patients requiring lymph node evaluation did not undergo a thorough lymph node evaluation because of their general condition or personal preferences. Appropriate reconstruction methods were performed after surgical excision, including local flaps, STSG, and free flaps. Furthermore, we recommend adjuvant RT for all MCC patients. Finally, careful postoperative follow-up is needed to assess patients' prognosis.

This study has limitations due to the small number of patients, and further studies with more patients are required. Our study is nonetheless noteworthy as a contribution to the relatively scant literature on Asian MCC patients.

\section{NOTES}

\section{Conflict of interest}

No potential conflict of interest relevant to this article was reported.

\section{Ethical approval}

The study was approved by the Institutional Review Board of Pusan National University Hospital (IRB No. 1905-016-079) and performed in accordance with the principles of the Declaration of Helsinki. Written informed consents were obtained from each patient.

\section{Patient consent}

The patients provided written informed consent for the publication and the use of their images.

\section{Author contribution}

Conceptualization: Bae YC. Data curation: Bae YC, Lee YW. Formal analysis: Bae YC, Lee YW. Funding acquisition: Bae YC. Methodology: Bae YC, Kim HS. Project administration: all authors. Visualization: all authors. Writing - original draft: Lee YW. Writing - review \& editing: Bae YC, Lee YW. Approval of final manuscript: all authors.

\section{ORCID}

Yong Woo Lee https://orcid.org/0000-0001-8088-822X Yong Chan Bae https://orcid.org/0000-0002-0268-4667 Su Bong Nam https://orcid.org/0000-0002-9661-0879 Seong Hwan Bae https://orcid.org/0000-0002-7203-8978 Hoon-Soo Kim https://orcid.org/0000-0002-7649-1446

\section{REFERENCES}

1. Houben R, Schrama D, Becker JC. Molecular pathogenesis of Merkel cell carcinoma. Exp Dermatol 2009;18:193-8.

2. Miller RW, Rabkin CS. Merkel cell carcinoma and melanoma: etiological similarities and differences. Cancer Epidemiol Biomarkers Prev 1999;8:153-8.

3. Lanoy E, Costagliola D, Engels EA. Skin cancers associated with HIV infection and solid-organ transplantation among elderly adults. Int J Cancer 2010;126:1724-31.

4. Agbai ON, Buster K, Sanchez M, et al. Skin cancer and photoprotection in people of color: a review and recommendations for physicians and the public. J Am Acad Dermatol 2014;70:748-62.

5. Albores-Saavedra J, Batich K, Chable-Montero F, et al. Merkel cell carcinoma demographics, morphology, and sur- 
vival based on 3870 cases: a population based study. J Cutan Pathol 2010;37:20-7.

6. Fitzgerald TL, Dennis S, Kachare SD, et al. Dramatic increase in the incidence and mortality from Merkel cell carcinoma in the United States. Am Surg 2015;81:802-6.

7. Hodgson NC. Merkel cell carcinoma: changing incidence trends. J Surg Oncol 2005;89:1-4.

8. Saito A, Tsutsumida A, Furukawa H, et al. Merkel cell carcinoma of the face: an analysis of 16 cases in the Japanese. J Plast Reconstr Aesthet Surg 2009;62:1272-6.

9. Woo KJ, Choi YL, Jung HS, et al. Merkel cell carcinoma: our experience with seven patients in Korea and a literature review. J Plast Reconstr Aesthet Surg 2010;63:2064-70.

10. Song PI, Liang H, Wei WQ et al. The clinical profile of Merkel cell carcinoma in mainland China. Int J Dermatol 2012;51:1054-9.

11. Cassler NM, Merrill D, Bichakjian CK, et al. Merkel cell carcinoma therapeutic update. Curr Treat Options Oncol 2016; 17:36.

12. Eng TY, Boersma MG, Fuller CD, et al. A comprehensive review of the treatment of Merkel cell carcinoma. Am J Clin Oncol 2007;30:624-36.

13. Allen PJ, Bowne WB, Jaques DP, et al. Merkel cell carcinoma: prognosis and treatment of patients from a single institution. J Clin Oncol 2005;23:2300-9.

14. PDQ Adult Treatment Editorial Board. Merkel cell carcinoma treatment $\left(\mathrm{PDQ}^{\circledR}\right)$ : health professional version. PDQ Cancer Information Summaries [Internet]. Bethesda, MD: National Cancer Institute [cited 2019 Mar 23]. Available from: http://www.ncbi.nlm.nih.gov/books/NBK65713.

15. Allen PJ, Zhang ZF, Coit DG. Surgical management of Merkel cell carcinoma. Ann Surg 1999;229:97-105.

16. Bichakjian CK, Olencki T, Aasi SZ, et al. Merkel cell carci- noma, version 1.2018, NCCN clinical practice guidelines in oncology.J Natl Compr Canc Netw 2018; 16:742-74.

17. Perez MC, de Pinho FR, Holstein A, et al. Resection margins in Merkel cell carcinoma: is a $1-\mathrm{cm}$ margin wide enough? Ann Surg Oncol 2018;25:3334-40.

18. Boyer JD, Zitelli JA, Brodland DG, et al. Local control of primary Merkel cell carcinoma: review of 45 cases treated with Mohs micrographic surgery with and without adjuvant radiation. J Am Acad Dermatol 2002;47:885-92.

19. O’Connor WJ, Roenigk RK, Brodland DG. Merkel cell carcinoma: comparison of Mohs micrographic surgery and wide excision in eighty-six patients. Dermatol Surg 1997;23: 929-33.

20. Pitale M, Sessions RB, Husain S. An analysis of prognostic factors in cutaneous neuroendocrine carcinoma. Laryngoscope 1992;102:244-9.

21. Tarantola TI, Vallow LA, Halyard MY, et al. Prognostic factors in Merkel cell carcinoma: analysis of 240 cases. J Am Acad Dermatol 2013;68:425-32.

22. Prieto Munoz I, Pardo Masferrer J, Olivera Vegas J, et al. Merkel cell carcinoma from 2008 to 2012: reaching a new level of understanding. Cancer Treat Rev 2013;39:421-9.

23. Warner RE, Quinn MJ, Hruby G, et al. Management of Merkel cell carcinoma: the roles of lymphoscintigraphy, sentinel lymph node biopsy and adjuvant radiotherapy. Ann Surg Oncol 2008;15:2509-18.

24. Bischof M, van Kampen M, Huber P, et al. Merkel cell carcinoma: the role of radiation therapy in general management. Strahlenther Onkol 1999;175:611-5.

25. Lawenda BD, Arnold MG, Tokarz VA, et al. Analysis of radiation therapy for the control of Merkel cell carcinoma of the head and neck based on 36 cases and a literature review. Ear Nose Throat J 2008;87:634-43. 